\section{Multi-camera system of the endoscopy: endoscopic mucosal resection for large gastric lesion using a novel 1-channel camera-hood}

Endoscopic procedures, such as endoscopic mucosal resection (EMR) for large gastric lesions, are performed blindly [1, 2]. Precise snaring during EMR is important to carry en-block resection. However, this can be difficult to achieve in practice because it is impossible to perform snaring by complete observation. Although we can easily observe the proximal side of the lifting lesion, it is hard to see the distal side after injection of saline solution into the submucosa. Therefore, we developed a novel 1-channel camerahood, which allows an observation of the distal side of the lesion during snaring in EMR procedures.

Recently, we developed a novel one-third partial transparent hood that facilitates endoscopic hemostatic procedures, while simultaneously allowing irrigation of the bleeding site [3]. The one-third partial hood is easily placed on the tip of the endoscope, although the hood has to be fitted to the right side of the endoscope (Figure 1). The 1-channel camera-hood was fabricated by cutting the partial hood into a 'U-shape' in the cap portion of the hood, and then attaching a machined camera for dental use, which consisted of charge coupled device (CCD) camera and four light-emitting diodes (LED) ("Miharu-kun"; RF System Lab., Japan) through two tubes (Figure 2 ). The length of the two tubes is variable, and one of these is an accessory channel.

The EMR procedure using the 1-channel camera-hood was performed as follows (Figure 3). Marking dots were made on the circumference of the target tumor to outline the margin. After injection of a saline solution into the submucosa, the tumor was separated from surrounding normal mucosa by complete incision around the lesion using the insulated-tip knife. The endoscope was then removed, and the 1-channel camera-hood was placed on the tip and fixed with tape. A snare was passed through the accessory channel of the hood, and a grasping forceps was passed through the accessory
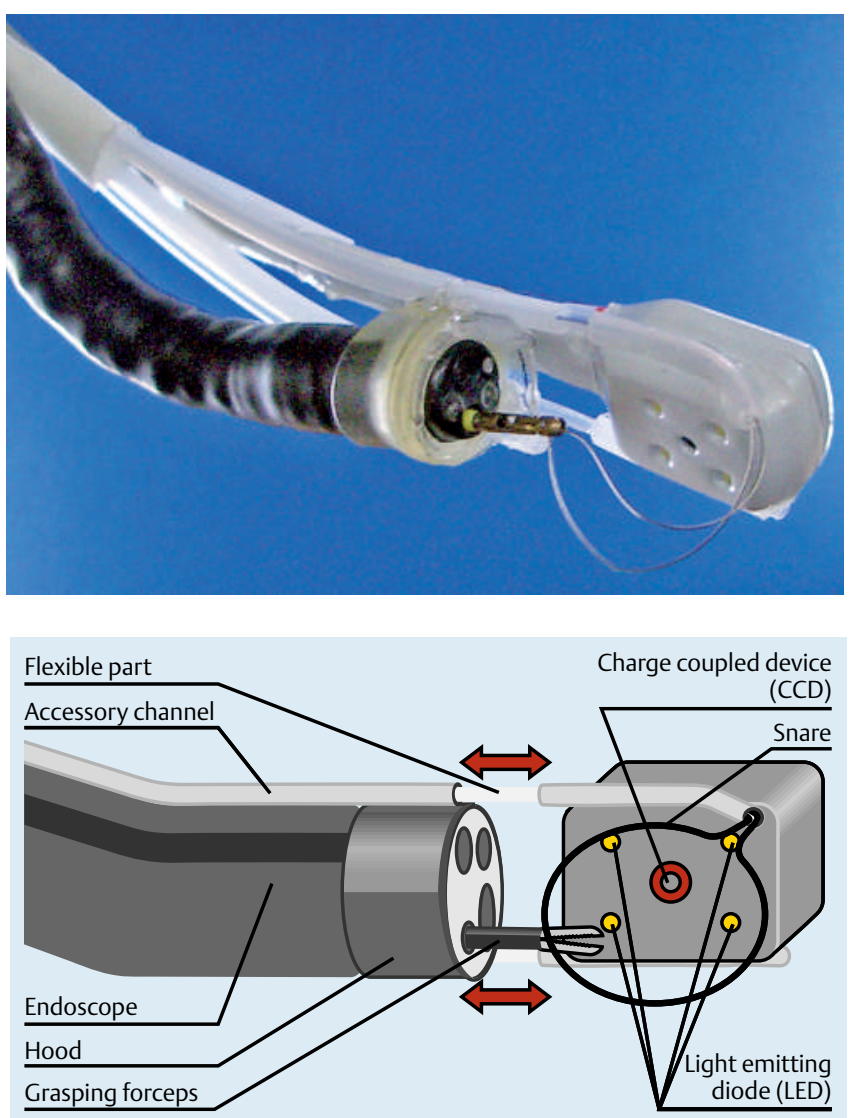
hood.

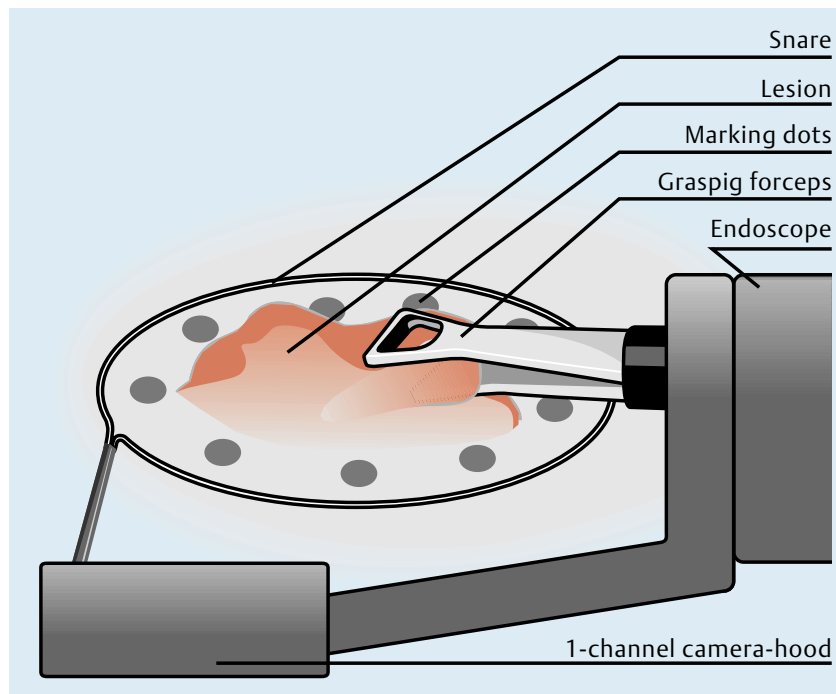

channel of the endoscope. We made the grasping forceps catch hold of the snare. We strangulated the lesion by precisely closing the snare, while allowing adequate observation by both $\mathrm{CCD}$ cameras of the 1-channel camera-hood and the en-

Figure 1 The 1-channel camera-hood being placed at the tip of the endoscope.

Figure 2 Schema of the 1-channel camera-

Figure 3 Schematic representation of endoscopic mucosal resection using the 1channel camera-hood.

doscope. Blend electrosurgical current was used to resect the lesion. 
Two specimens were resected in an animal model (pigs). The average diameter of the resected specimen was $30 \mathrm{~mm}$.

Endoscopy_UCTN_Code_TTT_1AO_2AG

\section{K. Kume, M. Yamasaki, I. Yoshikawa, M. Otsuki}

The Department of Gastroenterology and Metabolism, University of Occupational and Environmental Health, Japan, School of Medicine, Kitakyushu, Japan.
References

${ }^{1}$ Ono H, Kondo H, Gotoda T et al. Endoscopic mucosal resection for treatment of early gastric cancer. Gut 2001; 48: 225-229

2 Ohkuwa M, Hosokawa K, Boku N et al. New endoscopic treatment for intramucosal gastric tumors using an insulated-tip diathermic knife. Endoscopy 2001; 33: 221 - 226

${ }^{3}$ Kume K, Yamasaki M, Yamasaki T et al. Endoscopic hemostatic treatment under irrigation for upper GI hemorrhage: a comparison of one third and total circumference transparent end hoods. Gastrointest Endosc 2004; 59: $712-716$
Corresponding author

\section{K. Kume, MD}

The Department of Gastroenterology and Metabolism

University of Occupational and Environmental Health, Japan

School of Medicine

1-1, Iseigaoka

Yahatanishi-ku

Kitakyushu 807-8555

Japan

Fax: $\quad$ +81-93-692-0107

Email: mac-otsk@med.uoeh-u.ac.jp 